\title{
Quantitative growth and development of human brain
}

\author{
JOHN DOBBING and JEAN SANDS \\ From the Department of Child Health, University of Manchester, Manchester
}

\begin{abstract}
Dobbing, J., and Sands, J. (1973). Archives of Disease in Childhood, 48, 757. Quantitative growth and development of human brain. One hundred and thirty-nine complete human brains ranging in age from 10 weeks' gestation to 7 postnatal years, together with 9 adult brains, have been analysed in order to describe the human brain growth spurt quantitatively. The three major regions were examined for weight, DNA, cholesterol, and water content. The growth spurt period is much more postnatal than has formerly been supposed. The cerebellum has special growth characteristics; and there is a separate period from 10 to 18 weeks' gestation when adult neuronal cell number may largely be achieved. The implications of these findings for the vulnerability of developing brain are discussed.
\end{abstract}

The transient period of the brain growth spurt has been shown to be one of special vulnerability to the consequences of nutritional growth restriction. Certain deficits and distortions of adult brain structure and function have been produced in experimental animals by quite moderate growth restriction imposed at this stage of development (Dobbing and Smart, 1973). It is likely that the brain growth spurt is also a period of additional vulnerability to disorders other than undernutrition, such as inborn errors of metabolism, $x$-irradiation, and endocrine imbalance. It is even possible that the successful accomplishment of a normal brain growth spurt under good conditions may be a prerequisite for satisfactory subsequent bodily growth.

The evidence for these propositions inevitably comes from animal experiment, and the important question therefore arises whether such findings can reasonably be extrapolated from one species to another, and especially from laboratory animals to man.

It has been argued (Dobbing, 1970a, 1973) that meaningful interspecies extrapolation in this field depends heavily on taking into account the timing of birth in relation to the brain growth spurt. This timing varies from one species to another, according to the maturity of the species at birth. In other words, the brain of one developing species must be compared with that of another at the same developmental stage, regardless of whether this be fetal, perinatal, or postnatal. Successful extrapolation, therefore, depends on a knowledge of the

Received 12 April 1973. timing of the brain growth spurt in the species concerned as well as attention to the severity, nature, and duration of any restrictions in relation to the growth spurt period.

For these reasons it has become necessary to delineate the normal human brain growth spurt in quantitative terms comparable with those already described in experimental animals. The present paper attempts to do this.

\section{Material}

Selection. 139 complete, 'normal' human brains were collected, ranging in age from 10 weeks' gestation to 7 postnatal years, together with 9 'adult' brains to represent the target of adult growth achievement.

The normality of the sample was ensured as far as possible, partly by the method of selection of specimens, and partly by restricting measurements to parameters which are not significantly affected by the inevitable interval between death and availability.

All fetal ages were calculated from the first day of the last menstrual period. The selection of specimens was different in each of four age ranges. (1) From 10 to 22 weeks' gestation brains came from 29 fetuses aborted for nonpathological reasons, which may therefore be presumed normal. (2) From 25 weeks' gestation to term 36 brains were selected from the necropsy room population on the basis (a) of their body weights being within $1 \mathrm{SD}$ of that expected for the gestational age and (b) of there being no conspicuous neuropathology. (3) From birth to 7 years the 65 brains came from children who had suffered accidental death or had died acutely from conditions not conspicuously involving the nervous system. (4) An adult group of 9 brains ranging from 10 to 78 years of age and fulfilling the same criteria as in group 3. 
Dissection. Meninges and surface vessels were removed where this was easy, and the brains divided into three gross regions. Cerebellum was detached at its peduncles, and 'forebrain' separated from 'stem' by oblique cuts through the cerebral peduncles and transversely at the level of the mamillary bodies. Each part was then weighed and homogenized. An aliquot of the homogenate was sealed and stored at $-15^{\circ} \mathrm{C}$ to $-20^{\circ} \mathrm{C}$ for subsequent analysis.

Analysis. DNA was estimated by the method of Zamenhof et al. (1964) and cholesterol by the method of Leffler (1959). Water content was determined by drying at $99^{\circ} \mathrm{C}$ to constant weight. Neither the DNA nor the cholesterol content of a freshly killed animal brain was affected by storage at room temperature for a week. Whole part DNA and whole part cholesterol were calculated by multiplying the concentration per unit fresh weight in that part by its total weight. Whole brain total values were the sum of the whole part total values. Whole brain concentrations were then calculated accordingly. Water was expressed as a percentage of the fresh weight.

Indices. Whole part DNA was used as an index of total cell number on the assumption that the overwhelming majority of brain cells were diploid. DNA concentration was likewise regarded as an index of 'cellularity'. Cholesterol concentration was used as some index of myelination as previously described (Davison and Dobbing, 1968).

\section{Results}

Body weight and brain weight. The body weights of the 118 babies comprising the sample up to 14 postnatal months of age are shown in Fig. 1. Also shown are curves representing 1 and 2 SD

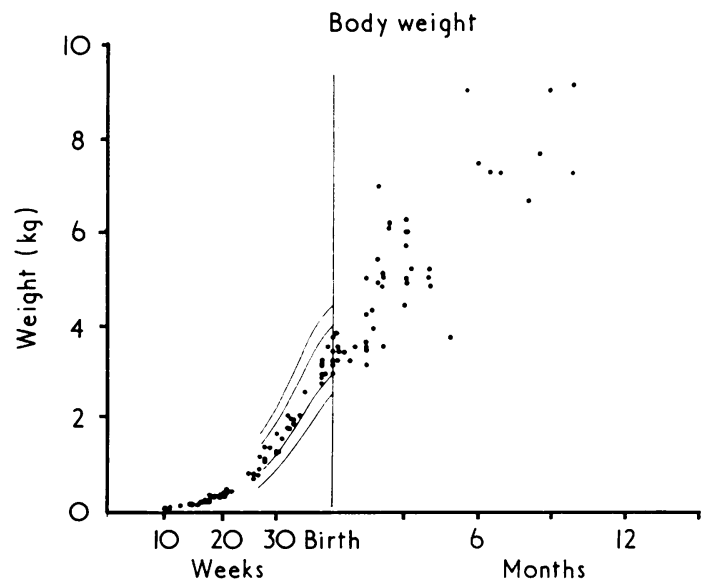

FIG. 1.-Prenatal and postnatal body weights of the early part of the sample from 10 gestational weeks to 12 postnatal months. The double lines from 27 gestational weeks to term represent 1 and $2 S D$ from the expected weight for gestational age (see text). from the expected weight for gestational ages between 28 and 40 weeks (Butler and Alberman, 1969, Table $8 \cdot 1$ ). The few postnatal cases which do not appear to follow the expected shape of the curve have been retained in order to preserve the simple selection criteria described above.

Fig. 2a shows a definite, but comparatively slow
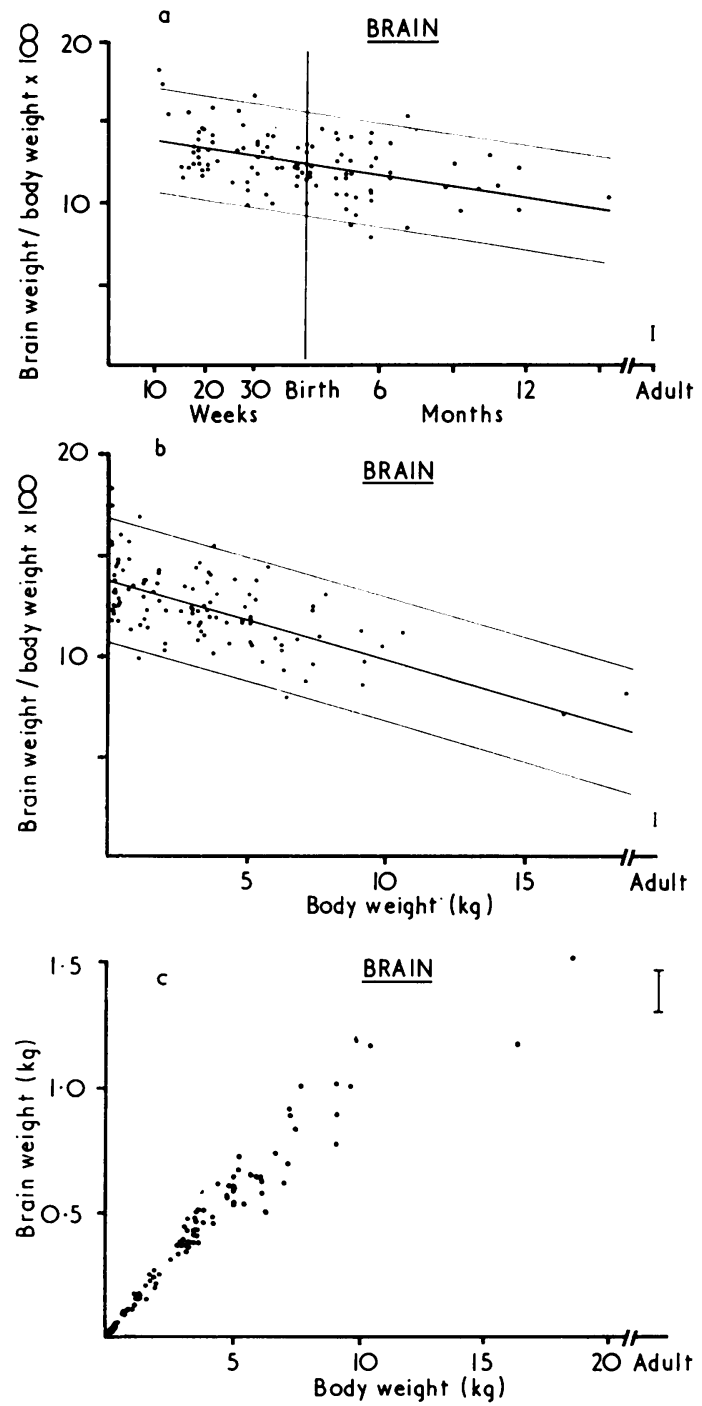

FIG. 2.-Weight of whole brain in relation to that of body. (a) Brain weight/body weight plotted against age. Brain weight/body weight ratio $\times 100=14 \cdot 3652-0.0476 \times$ conceptual age (weeks); tolerance limits $\pm 3 \cdot 33$. (b) Brain weight/body weight plotted against body weight. Brain weight/body weight ratio $\times 100=13.6435-0.3673 \times$ body weight $(\mathrm{kg})$; tolerance limits $\pm 3 \cdot 22$. (c) Brain weight plotted against body weight. 
fall of brain weight : body weight ratio with age, with a variance large enough to render this parameter valueless for predictive purposes. Some of the difficulties in establishing whether the ratio in any given dysmature baby is abnormal must also stem from the large size of this normal variance. Unfortunately, the usefulness of the ratio is not improved by plotting it against body weight (Fig. 2b). It is not clear (nor clinically very important) whether there is a sharp fall before 10 gestational weeks. The slow rate of fall illustrated in Fig. $2 a$ and $b$ is in marked contrast to that in pigs (Dickerson and Dobbing, 1967), guinea pigs (Dobbing and Sands, 1970a), and rats (Dobbing, 1968a). It may be more useful to plot brain weight directly against body weight as in Fig. 2c.

Fresh weights of the whole brain and its parts are shown in Fig. 3, with those for the stem showing a greater scatter than in other regions because of the comparative smallness of this part and the consequent greater effect of inconstancy of dissection. This applies to all subsequent results for the stem.

The postnatal cut-off point of the sigmoid curve of weight accumulation seems to be between 18 postnatal months and 2 years for whole brain and forebrain. It is a little earlier for cerebellum, a region which starts to grow later than the rest of the brain and reaches its plateau earlier. This characteristic of cerebellar growth is even more conspicuous in some of the parameters described below.

Cellularity and total cell number. These were measured by expressing the chemical estimation of DNA-P as a concentration/g fresh weight, and as total DNA-P/anatomical region, respectively. This use of DNA values depends on there being a constant amount of DNA per diploid nucleus, and
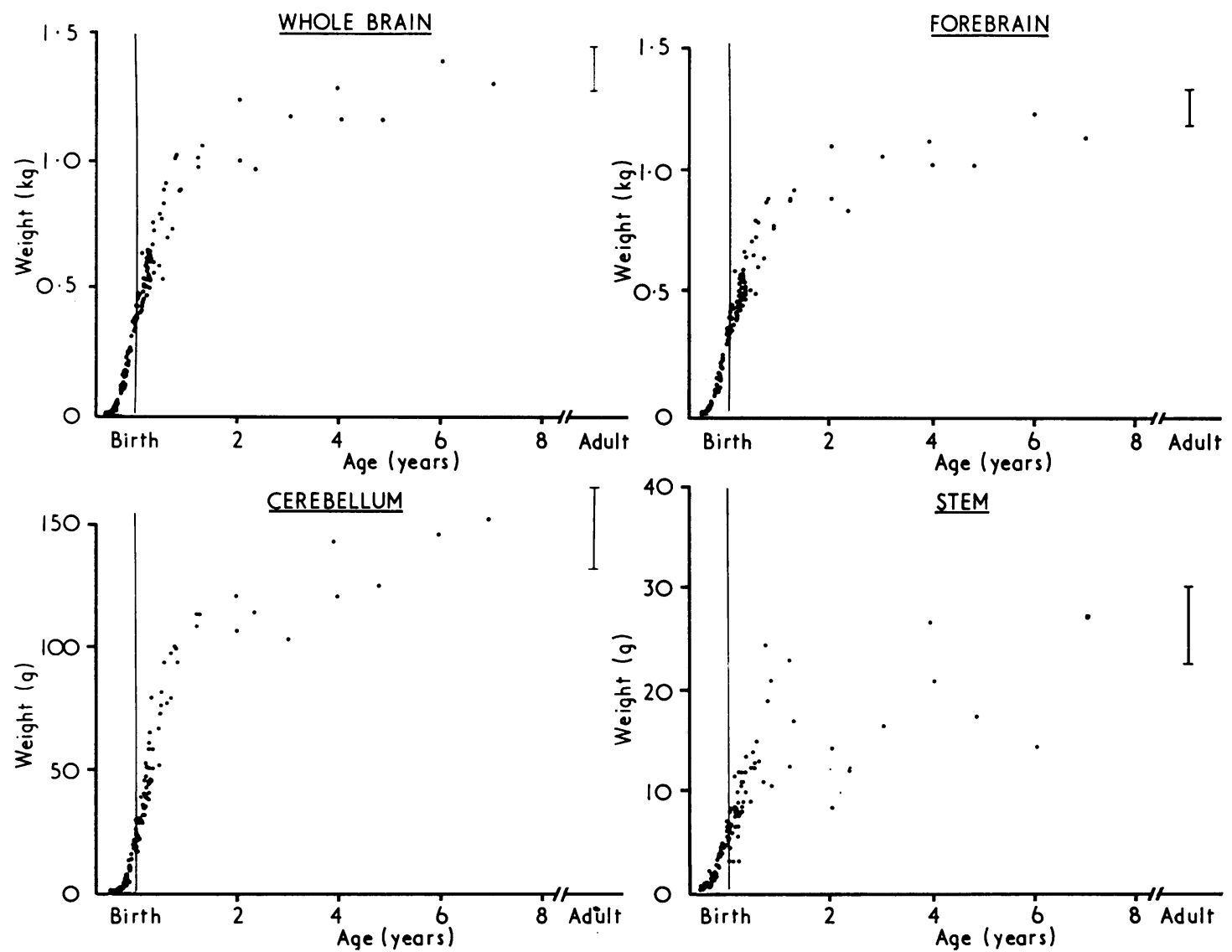

FIG. 3. Fresh weights of whole brain, forebrain, cerebellum, and stem during growth. 
on the number of nondiploid nuclei being negligible in relation to the total cell number (Dobbing and Sands, 1970a; Mann and Yates, 1973a, b). It also depends on the whole brain, or the whole anatomical region, being available for homogenizing. Representative samples such as biopsies are not acceptable in view of the histological heterogeneity of the brain tissue.

Cellularity (DNA/g tissue) is a difficult parameter to consider in growing brain, just as is the number of cells per microscope field, since in all parts except the cerebellum it falls with increasing age (Fig. 4) at the very time when the total number of cells is rising. Whole brain cellularity also falls because of the quantitative dominance of the forebrain. The fall is due to several other parameters of growth which have a net diluting effect on cell number. Thus the growth in weight outpaces the growth in numbers of cells because of the disproportionately rapid growth in cell size, dendritic branching, and myelination at this time. Once again the cerebellum is the exception. Its cellularity increases when it is falling elsewhere, presumably due partly to its extraordinarily rapid rate of cell multiplication (see below) and partly to the comparatively small ultimate size of most of its cells.

Total cell number is a more meaningful parameter, but also carries the practical disadvantage that the whole brain, or the whole region must be available for estimation, so that it can be homogenized. The total DNA-P is shown in Fig. 5. In whole brain, the cut-off point marking the levelling off point of the sigmoid curve seems to begin at about 18 postnatal months, similar to that in the weight curve (Fig. 3, whole brain). Some of its sharpness of definition is, however, attributable to the inclusion of the cerebellum in the calculation,
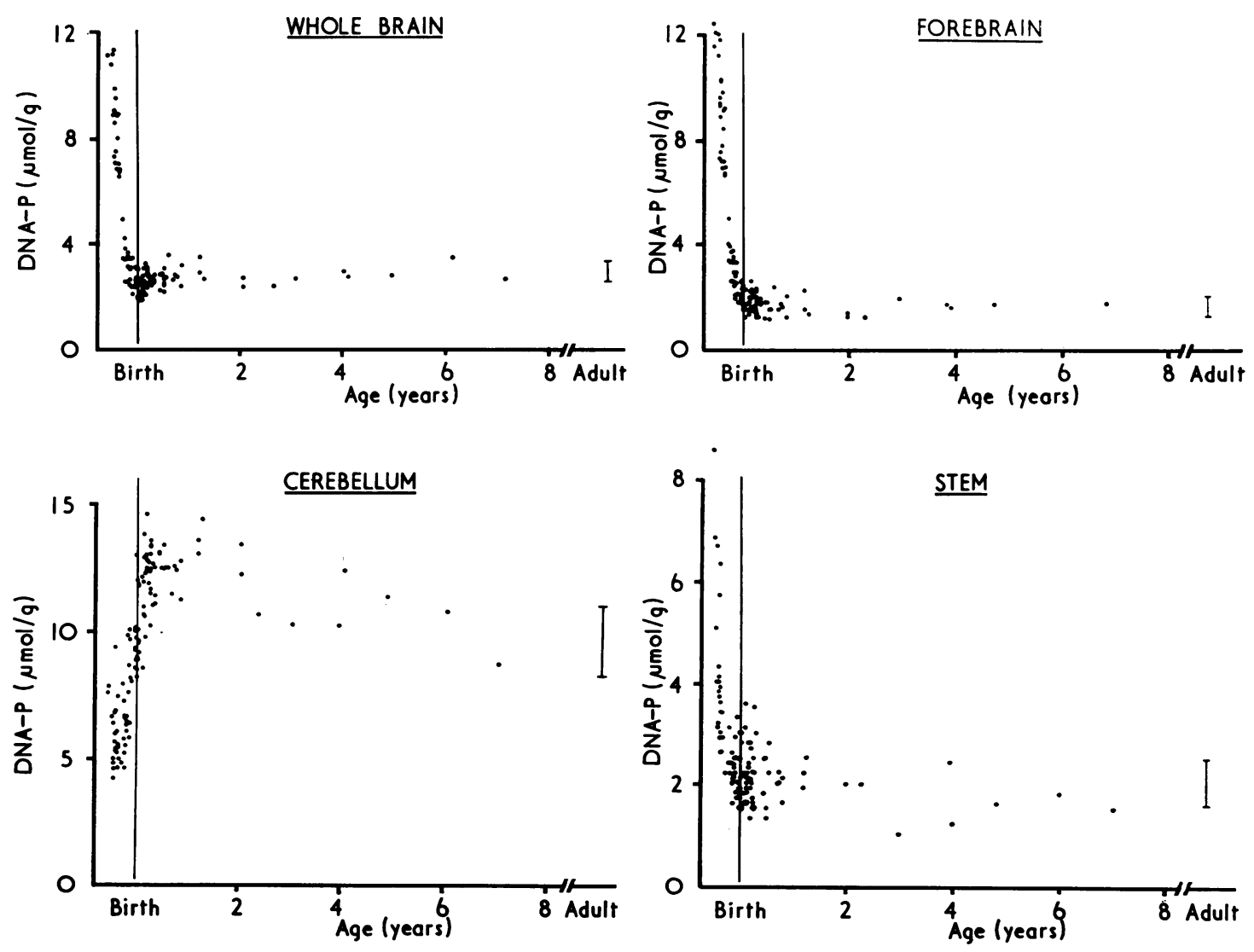

FIG. 4.-Concentration of DNA-P/unit fresh weight, equivalent to cellularity (see text), in whole brain, forebrain, cerebellum, and stem during growth. Compare with Fig. 5. 
which brings the cut-off point forward a little. Total DNA-P in the cerebellum shows more clearly than any other parameter how the cerebellum begins to grow later, but finishes earlier, than the rest of the brain, and must therefore grow much faster. Also, though it weighs little more than oneeighth of the forebrain, it contains as much as half as many cells.

When the curve for total forebrain DNA-P is examined separately, the cut-off point is less clear, but is probably even later than that found for whole brain. One of the difficulties in deciding more precisely when it occurs is the shortage of specimens older than 18 months. Another is that the end-point is also less sharp. However, it probably occurs not much before 2 postnatal years; and this phase of total cell multiplication occupies only the early part of the entire brain growth-spurt complex.

Neurons and glia. One difficulty in using DNA-P values as an index of cell number is that it fails to distinguish between different cell types. However, in one respect it is likely that neuronal can be distinguished from glial multiplication since these are largely consecutive processes (Peters and Flexner, 1950). In small laboratory species, due to their much shorter developmental time-scales
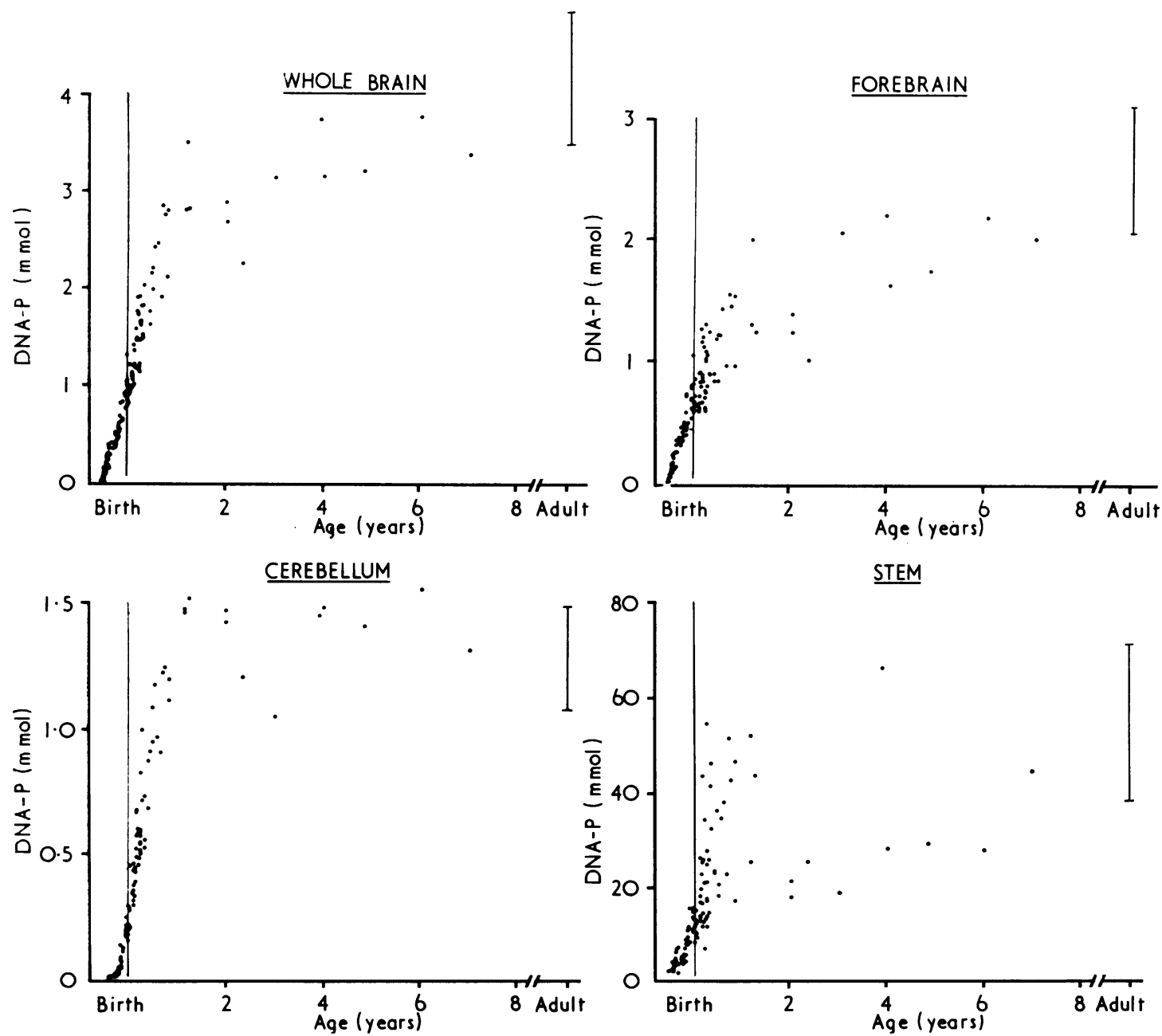

FiG. 5.-Total DNA-P, equivalent to total cell number, in whole brain, forebrain, cerebellum, and stem during growth. Compare with Fig. 4. 
and the difficulty of timing their pregnancies to within fractions of a day, the expected two phases of neuronal followed by glial multiplication are difficult to distinguish. The much slower developmental rate in humans and the opportunity to examine a sufficient number of specimens of the appropriate age has revealed these two phases in human forebrain, as illustrated in Fig. 6. The logarithmic expression in Fig. $6 \mathrm{~b}$ shows a particularly sharp angulation of the curve at 18 weeks' gestation, much sharper and perhaps earlier than
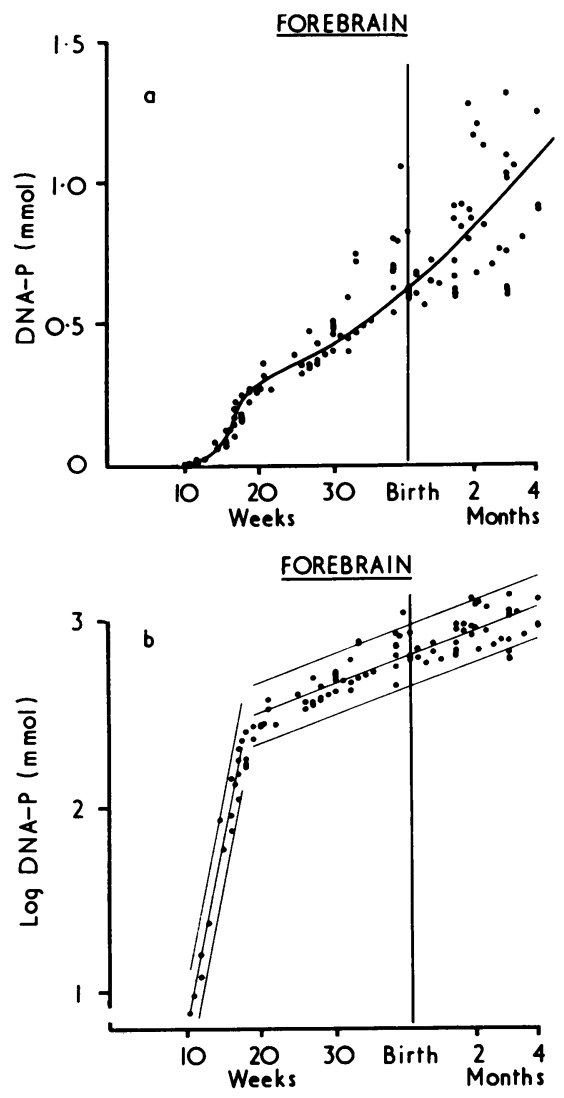

FIG. 6.-(a) Total DNA-P, equivalent to total cell number, in the forebrain from 10 gestational weeks to 4 postnatal months, showing the two-phase characteristics of prenatal cell multiplication. (b) A semilogarithmic plot of the data appearing in (a) to show the comparatively sharp separation of the two phases at 18 gestational weeks. Regression lines with $95 \%$ confidence limits are added. First phase: log forebrain DNA-P $P_{10-17.5}(\mathrm{mmol})=$ $0.2051 \times$ conceptual age (weeks) -1.2748; tolerance limits $\pm 0 \cdot 25$. Second phase: log forebrain $D N A-P_{19-57}$ $(\mathrm{mmol})=0.0149 \times$ conceptual age (weeks) +2.1984 ; tolerance limits $\pm 0 \cdot 19$. previously published (Dobbing and Sands, 1970b), when fewer specimens were available. If this is a correct interpretation of the two phases of cell multiplication, it does not, of course, imply that adult numbers of neurons are completely present at 18 weeks. It is known that some divide later, and many do not survive. Nevertheless, the laterdividing neuroblasts seem not to influence the broad picture illustrated in Fig. $6 \mathrm{~b}$.

Myelination. The advantages of using cholesterol as an index of myelination are its ease of estimation and its chemical stability in postmortem tissue. However, it is a much less specific myelin lipid than some, since it is present in all other cell membranes besides myelin. In all animal species studied it accumulates a little before the deposition of more specific myelin lipids, and its use is therefore likely to underestimate the duration of the rapid phase of true myelination. There are at present no satisfactory morphological methods for measuring rate or degree of myelination, and so the use of chemical indices is extremely useful.

Cholesterol, like DNA-P, may be expressed as total amount per anatomical region, or as a concentration per unit weight. The meaning of the two expressions has been discussed elsewhere (Davison and Dobbing, 1968). It has been suggested that the process of myelination may have two overlapping components: a 'maturational' one consisting of gradual thickening of the laminated sheaths around existing lengths of axon and a 'growth' process consisting of myelination concurrent with growth in axonal length. The former will mainly contribute to an increase in cholesterol per unit wet weight, since the lipid deposition occurs at the expense of water (see below). The latter will contribute little to the increase of lipid concentration, since fresh weight will be increased along with lipid. It will, however, add to the total lipid per region. Thus, in this general sense, myelination as a developmental, maturational process will be more reflected by increased lipid concentration; and the concurrent and later myelination of growth will be reflected by increased total amount.

In all animal species studied the phase of lipid deposition is later than that of cell multiplication. This part of the developmental sequence appears to be due to the necessity to establish an oligodendroglial population before these same cells can synthesize the myelin (Davison and Dobbing, 1968).

Cholesterol concentration and total cholesterol are shown in Fig. 7 and 8, respectively. In both cases the faster growth performance of the cerebellum can again be discerned, comparable with the 

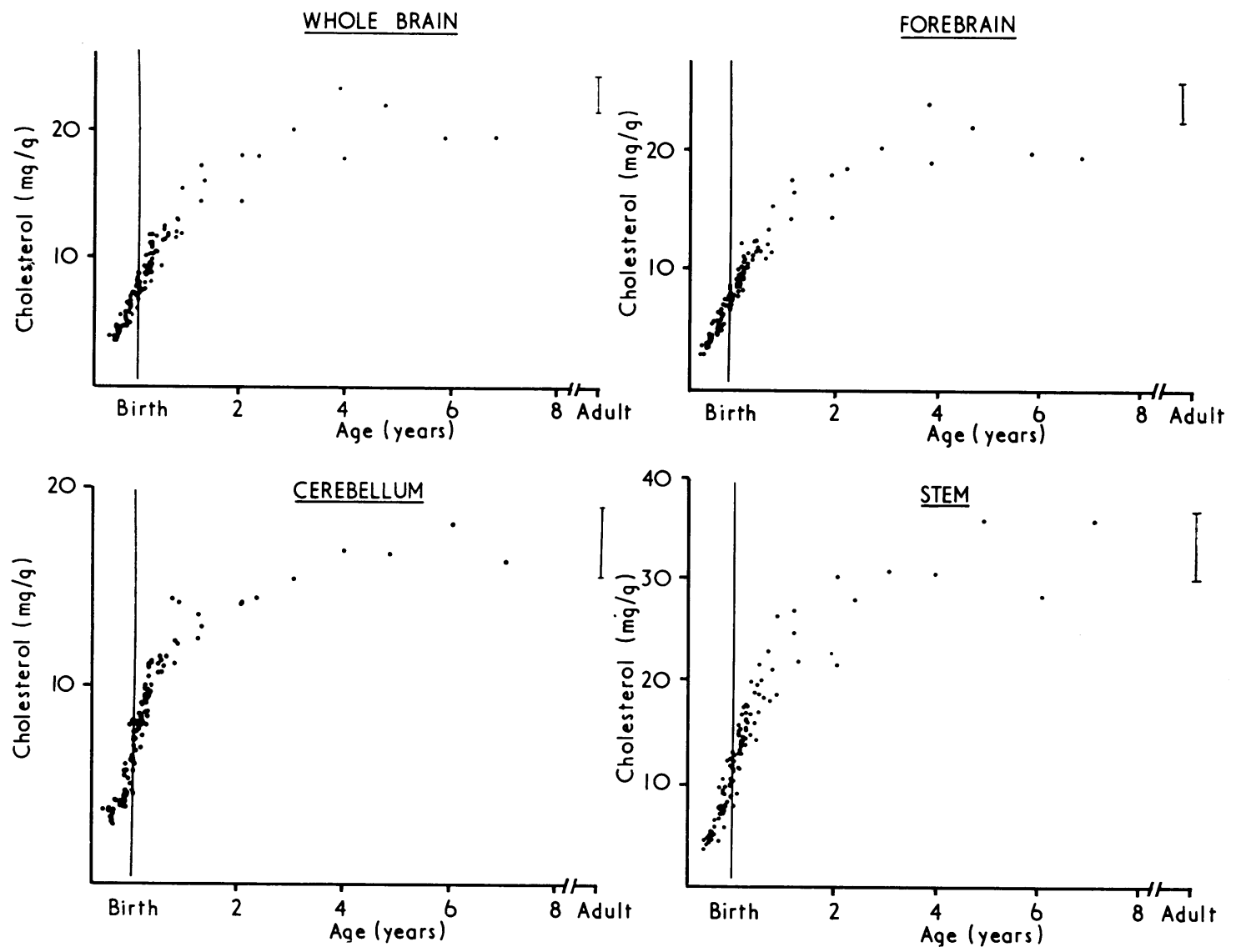

FIG. 7.-Concentration of cholesterol/unit fresh weight in whole brain, forebrain, cerebellum, and stem during growth (see

Fig. 8).

foregoing results for other parameters. The endpoint of the growth spurt is less sharply defined, but, as would be expected from a similar sequence in all other animals, it is later than that for cell multiplication, perhaps extending in the whole brain, forebrain, and stem to 3 or 4 postnatal years. In view of the even later appearance of the more specific myelin lipids, the growth-spurt complex, including myelination, may possibly continue into the 4 th or 5 th postnatal year.

Water content. The brain growth spurt in all species is a period of rapidly declining water content. The falling curve of water concentration remarkably resembles the reciprocal of the rising lipid concentration. That the same is true of the developing human brain can be seen in Fig. 9. The fact that the fall in water is so precipitous in the perinatal period makes the diagnosis of perinatal cerebral oedema almost impossible even by water estimation in the laboratory, and it is inconceivable that even the most experienced pathologist will be able to make a diagnosis of oedema by means of a subjective estimation of wetness alone. The relatively high rapidity of loss of cerebellar water is in keeping with its previously noted growth characteristics.

Relative growth of brain regions. Throughout this paper the temptation to draw subjective, smoothed lines through the data has so far been resisted. However, it would have been impossible to represent the relative growth of the three gross regions without doing so. Fig. 10 and 11 show subjective assessments of growth in weight and number of cells, respectively, of forebrain, cerebellum, and stem. In both figures the differentially 

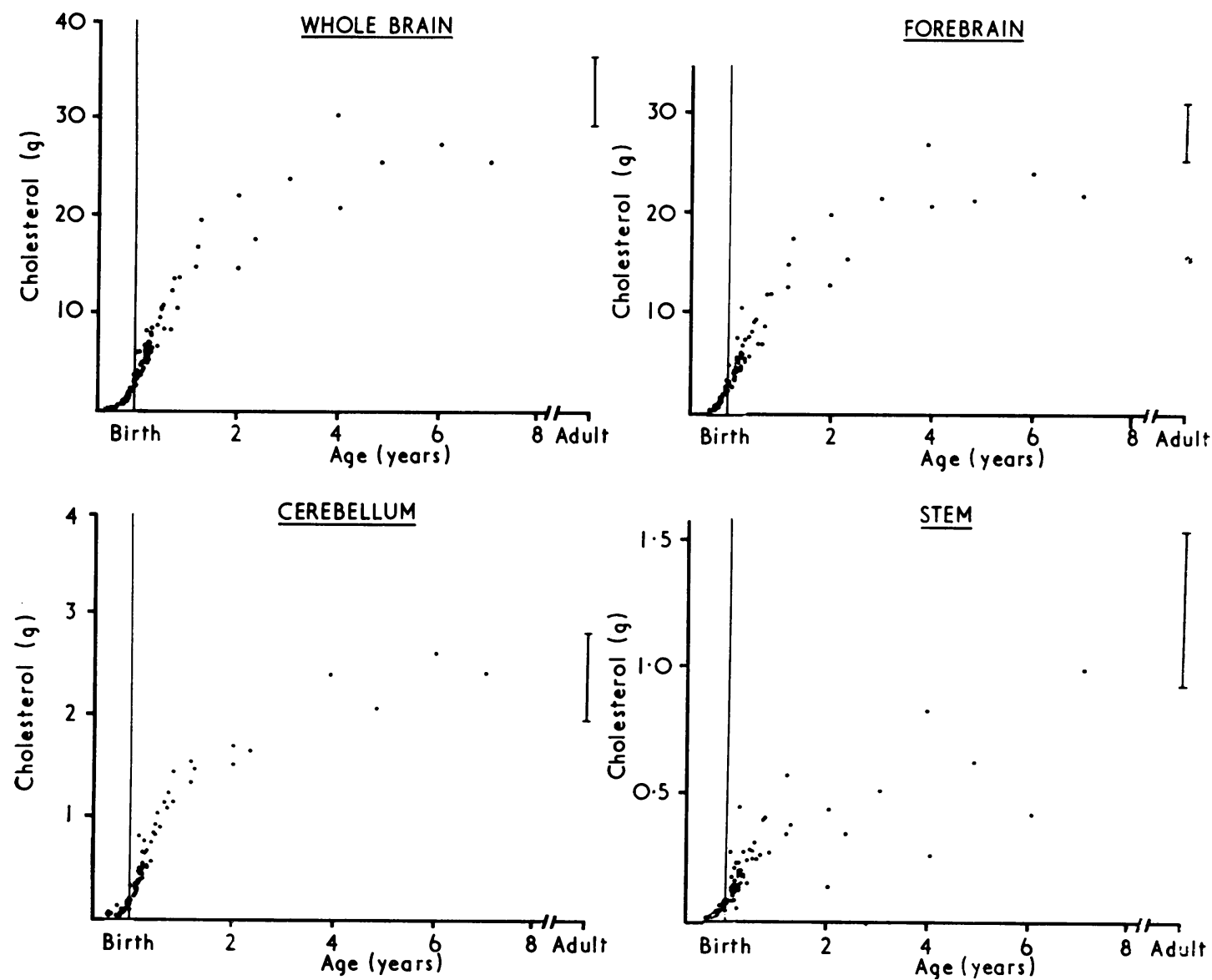

FIG. 8.-Total cholesterol in whole brain, forebrain, cerebellum, and stem during growth (see Fig. 7).

more rapid growth of the cerebellum can be seen. Fig. 11 shows that, as a result of an extraordinarily rapid rate of cell multiplication adult numbers of cells are present in the cerebellum before the age of 18 postnatal months, at a time when the forebrain and stem are barely $60 \%$ of the way towards their target. It should be noted that the cerebellum does not grow later than the rest of the brain as is often stated. It starts later, but it finishes earlier, and grows much faster throughout its shorter growth spurt.

\section{Discussion}

The main purpose of the present study was to identify the outline of the human brain growth spurt equivalent to that which has been shown to be particularly vulnerable to growth restriction in experimental animals (Dobbing, 1968b, 1972). Individual aspects of the findings have already been discussed above.

The brain growth spurt was originally defined as the transient period of rapid brain growth which can be seen in all species. In gross anatomical terms it begins when the adult neuronal number has already been largely achieved, except perhaps for certain cerebellar neurons. Thus it begins at about birth in the rat and about midgestation in the human. The enormous multiplication of cells which occupies its early part is, therefore, predominantly glial. The most easily measured component of the later phases of the growth spurt is myelination which has awaited the establishment of a glial cell population. In the rat the phase of rapid myelination, and hence the arbitrarily defined brain 

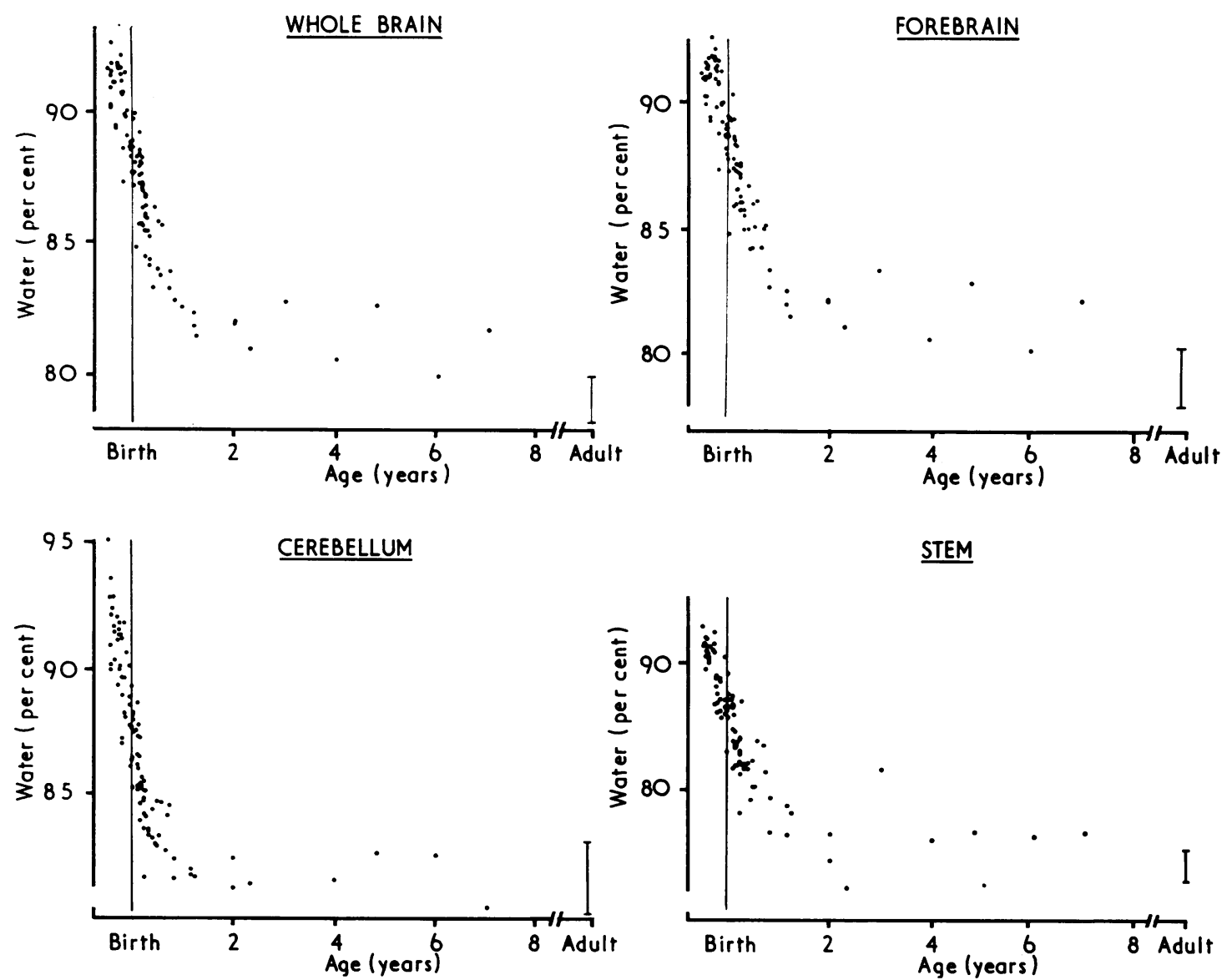

FIG. 9.-Percentage of water in whole brain, forebrain, cerebellum, and stem during growth.

growth spurt is ended by about 25 postnatal days, a stage reached by the human brain by about 3 to 4 postnatal years.

It is important to appreciate that though the phase of neuronal multiplication precedes this arbitrarily defined vulnerable period, the tremendous increase in dendritic complexity accompanied by the establishment of synaptic connectivity must inevitably be later, and almost certainly coincides and is coextensive with it. The more important of the observed consequences of growth restriction at this time may therefore be more related to interference with the latter processes (which we cannot at present easily measure) than to the reduction in glial cell number and myelination (which we can).

This is not to say that interference with the pre-growth spurt phase of neuroblast multiplication is unimportant: but merely that in humans it occurs at a more highly protected early period of gestation: this is in contrast with the rat in which neuronal multiplication occurs in the last trimester of pregnancy, and for that reason alone is exposed to the hazards of maternal malnutrition. It is not likely in humans that fetal growth restriction results from maternal malnutrition in the early second trimester. This illustrates one of the pitfalls in extrapolating from rat fetal brain growth restriction to human (Dobbing, 1973). The difference between species is entirely one of timing, and it operates in favour of species with earlier, more protected neuronal multiplication. However, if the effects of malnutrition on the growth and establishment of synaptic connexions are more important than marginal effects on neuronal number (and this seems likely), the human is very little more protected than the rat, since in both 


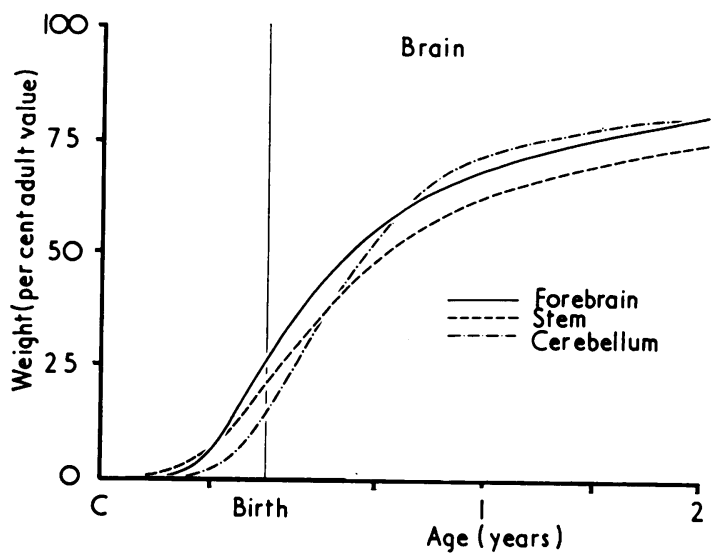

FIG. 10.-Comparative fresh weights of 3 brain regions during growth. Weights shown in Fig. 3 for forebrain, cerebellum, and stem have been calculated as a percentage of adult value, and smooth lines drawn by eye through the points.

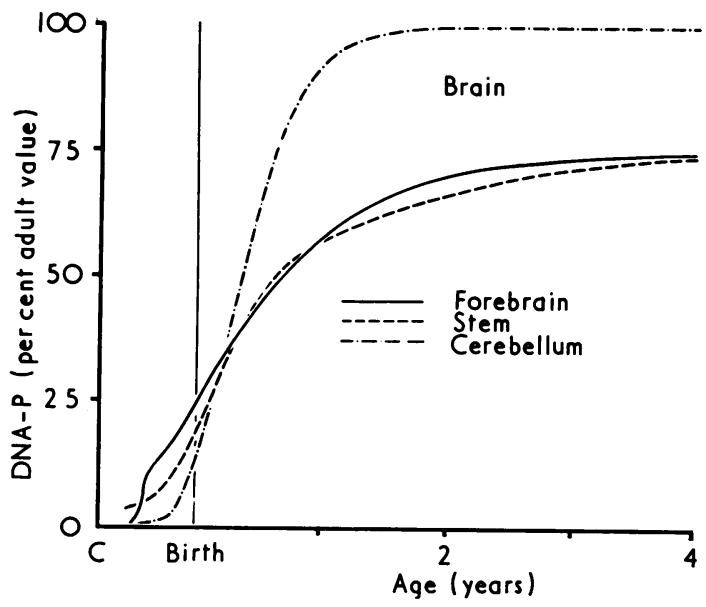

FIG. 11.-Comparative values for total DNA-P, equivalent to total numbers of cells (see text), in 3 brain regions. Values shown in Fig. 5 for forebrain, cerebellum, and stem have been calculated as a percentage of adult value, and sinooth lines drawn through the points.

species this phase of brain growth is predominantly postnatal. In the rat it occupies the first 25 postnatal days. In the human it runs from midpregnancy into the second postnatal year, or even well beyond.

The demonstration that human neuroblasts appear to multiply mainly between 10 and 18 weeks' gestation leads to a consideration of grossly abnormal circumstances which may possibly affect the fetus even at that early time. There is, for example, a striking similarity between this data and the incidence of microcephaly and mental retardation in children exposed as fetuses to the Hiroshima and Nagasaki bombs (Miller and Blot, 1972). The high incidence of this subnormality was largely confined to fetal exposure at 18 weeks or before, and though the similarity may be fortuitous, it could also presumably be causal. Viral infection at this time, leading to microcephaly, as well as chromosomal anomalies, $x$-irradiation, and steroid or other therapy may also be considered hazardous to neuronal division; and it may even be worth investigating whether some cases of 'unclassified' mental retardation may not be suffering from quantitative consequences of interference with this earlier phase of brain growth. Finally, the later brain growth spurt period may well be the main period of vulnerability to the ill-effects of inborn errors of metabolism. Though dietary therapy of these conditions must completely cover the whole period, it is possible that it could be relaxed afterwards with little untoward effect. Such a conclusion will only be established by careful observation of cases known to be defaulting in their dietary care after the first few years.

The peculiarly rapid rate of growth of the human cerebellum over a shorter but coincident period of the brain growth spurt has been mentioned several times in this account. The same characteristics are found in all animal species examined (Dickerson and Dobbing, 1967; Dobbing, Hopewell, and Lynch, 1971). It is known that this is associated in rats with differential vulnerability of the cerebellum to nutritional growth restriction (Dobbing, $1970 \mathrm{~b}$ ) and to postnatal $x$-irradiation (Dobbing et al., 1970); and an associated clumsiness has also been shown (J. Dobbing, A. Lynch, and J. L. Smart, in preparation). It seems reasonable that if vulnerability is related to rate of growth, the part of the brain which is growing fastest will show the greatest effects of growth restriction. The question whether the human cerebellum is ever meaningfully affected in this way is quite unresolved, and will remain so as long as pathologists confine their attention to lesion pathology and gross morphological anomaly. As an alternative approach, it would probably be useful at first merely to weigh the cerebellum routinely in relation to the weight of the rest of the brain in cases of growth retardation during the cerebellar growth spurt.

It has long been known that different species are born at differing stages of maturity. It was not difficult to express this as different timing of the brain growth spurt in relation to birth. Thus the 
rat (Dobbing and Sands, 1971) was characterized as a postnatal, the guinea-pig (Dobbing and Sands 1970a) a prenatal, and the pig (Dickerson and Dobbing, 1967) as a perinatal brain developer. It was then considered that the human brain growth spurt was perinatal like that of the pig. It was not, however, realized that though it began in midpregnancy, it continued well into the second postnatal year and even beyond, since it had previously been stated that the phase of cell division in human brain was finished by about 5 postnatal months (Winick, 1968). It now appears that at least $5 / 6$ of the human brain growth spurt is postnatal, and that in this respect humans resemble rats much more closely than was formerly thought. In practical terms this may offer us a much better postnatal opportunity to promote human brain growth by establishing the best environmental conditions at this important time.

We are grateful to our pathologist colleagues, Drs. A. J. Barson, N. J. Brown, and Professor F. A. Langley, without whose co-operation this study would not have been possible; and also gratefully acknowledge grants from the Medical Research Council and the National Fund for Research into Crippling Diseases, of Great Britain.

\section{REFERENCES}

Butler, N. R., and Alberman, E. D. (1969). (Editors) Perinatal Problems, p. 142. Livingstone, London.

Davison, A. N., and Dobbing, J. (1968). The developing brain. In Applied Neurochemistry, p. 253 . Ed. by A. N. Davison and J. Dobbing. Blackwell, Oxford.

Dickerson, J. W. T., and Dobbing, J. (1967). Prenatal and postnatal growth and development of the central nervous system of the pig. Proceedings of the Royal Society B, 166, 384.

Dobbing, J. (1968a). Effects of experimental undernutrition on development of the nervous system. In Malnutrition, Learning and Behaviour, p. 181. Ed. by N. S. Scrimshaw and J. E. Gordon. Massachusetts Institute of Technology, Boston.

Dobbing, J. (1968b). Vulnerable periods in developing brain. In Applied Neurochemistry, p. 287. Ed. by A. N. Davison and J. Dobbing. Blackwell, Oxford.

Dobbing, J. (1970a). Undernutrition and the developing brain: the relevance of animal models to the human problem. American Fournal of Diseases of Children, 120, 411.
Dobbing, J. (1970b). Undernutrition and the develeping brain. In Developmental Neurobiology, p. 241. Ed. by W. A. Himwich. Thomas, Springfield, Illinois.

Dobbing, J. (1972). Vulnerable periods of brain development. In Lipids, Malnutrition and the Developing Brain, p. 8. Ed. by $\mathrm{K}$. Elliott and J. Knight. (Ciba Foundation Symposium jointly with the Nestlé Foundation.) Elsevier, Amsterdam.

Dobbing, J. (1973). The developing brain: a plea for more critical interspecies extrapolation. Nutrition Reports International, 7, 401.

Dobbing, J., Hopewell, J. W., and Lynch, A. (1971). Vulnerability of developing brain. VII. Permanent deficit of neurons in cerebral and cerebellar cortex following early mild undernutrition. Experimental Neurology, 32, 439.

Dobbing, J., Hopewell, J. W., Lynch, A., and Sands, J. (1970). Vulnerability of developing brain. I. Some lasting effects of X-irradiation. Experimental Neurology, 28, 442.

Dobbing, J., and Sands, J. (1970a). Growth and development of the brain and spinal cord of the guinea pig. Brain Research, $17,115$.

Dobbing, J., and Sands, J. (1970b). Timing of neuroblast multiplication in developing human brain. Nature, 226, 639.

Dobbing, J., and Sands, J. (1971). Vulnerability of developing brain. IX. The effect of nutritional growth retardation on the timing of the brain growth-spurt. Biology of the Neonate, $19,363$.

Dobbing, J., and Smart, J. L. (1973). Early undernutrition, brain development and behaviour. Clinics in Developmental Medicine, No. 47. Ethology and Development. Ed. by S. A. Barnett. Heinemann, London.

Leffler, H. H. (1959). Estimation of cholesterol in serum. American fournal of Clinical Pathology, 31, 310.

Mann, D. M. A., and Yates, P. O. (1973a). Polyploidy in the human nervous system. I. The DNA content of neurones and glia of the cerebellum. Fournal of the Neurological Sciences, 18, 183.

Mann, D. M. A., and Yates, P. O. (1973b). Polyploidy in the human nervous system. II. Studies of the glial cell populations of the Purkinje cell layer of the human cerebellum. fournal of the Neurological Sciences, 18, 197.

Miller, R. W., and Blot, W. J. (1972). Small head size after in utero exposure to atomic radiation. Lancet, $2,784$.

Peters, V. B., and Flexner, L. B. (1950). Biochemical and physiological differentiation during morphogenesis. VIII. Quantitative morphologic studies on the developing cerebral cortex of the fetal guinea pig. American fournal of Anatomy, 86, 133.

Winick, M. (1968). Changes in nucleic acid and protein content of the human brain during growth. Pediatric Research, 2, 352.

Zamenhof, S., Bursztyn, H., Rich, K., and Zamenhof, P. J. (1964). The determination of deoxyribonucleic acid and of cell number in brain. Fournal of Neurochemistry, 11, 505.

Correspondence to Dr. J. Dobbing, Department of Child Health, University of Manchester, Clinical Sciences Building, York Place, Manchester M13 0JJ. 